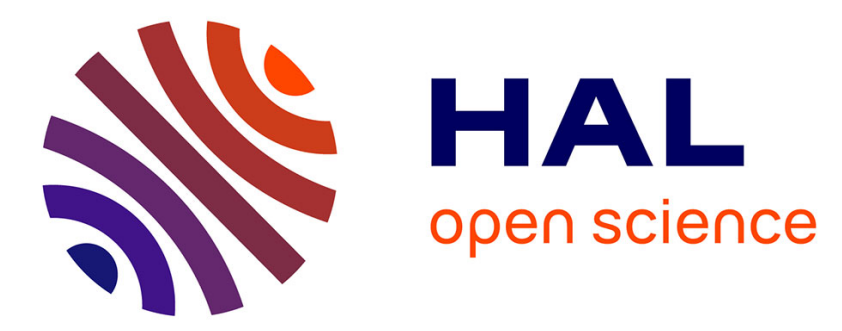

\title{
Fully frustrated simple cubic lattices and phase transitions
}

B. Derrida, Y. Pomeau, G. Toulouse, J. Vannimenus

\section{To cite this version:}

B. Derrida, Y. Pomeau, G. Toulouse, J. Vannimenus. Fully frustrated simple cubic lattices and phase transitions. Journal de Physique, 1980, 41 (3), pp.213-221. 10.1051/jphys:01980004103021300 . jpa00209236

\section{HAL Id: jpa-00209236 https://hal.science/jpa-00209236}

Submitted on 1 Jan 1980

HAL is a multi-disciplinary open access archive for the deposit and dissemination of scientific research documents, whether they are published or not. The documents may come from teaching and research institutions in France or abroad, or from public or private research centers.
L'archive ouverte pluridisciplinaire HAL, est destinée au dépôt et à la diffusion de documents scientifiques de niveau recherche, publiés ou non, émanant des établissements d'enseignement et de recherche français ou étrangers, des laboratoires publics ou privés. 


\title{
Fully frustrated simple cubic lattices and phase transitions
}

\author{
B. Derrida $(*)$ \\ Institut Laue-Langevin, 38042 Grenoble, France
}

\section{Y. Pomeau}

Service de Physique Théorique, CEN-Saclay, 91190 Gif sur Yvette, France

\author{
G. Toulouse and J. Vannimenus
}

Laboratoire de Physique de l'Ecole Normale Supérieure, 24 rue Lhomond, 75231 Paris Cedex 05, France

(Reçu le ler octobre 1979, accepté le 14 novembre 1979)

\begin{abstract}
Résumé. - Nous présentons l'expression explicite de l'énergie libre pour le modèle gaussien et le modèle sphérique sur un réseau cubique simple complètement frustré, en toutes dimensions, et comparons ces résultats avec le cas ferromagnétique. Pour le modèle sphérique, la température critique est deux fois plus basse que celle prédite par la théorie du champ moyen usuelle, tandis que l'approximation de Bethe ne prédit aucune transition. Nous étudions aussi le développement à haute dimension de l'énergie libre, et concluons que ce développement peut masquer les transitions. Ces résultats montrent que des difficultés rencontrées pour des modèles de verres de spin apparaissent aussi dans des modèles avec interactions périodiques.
\end{abstract}

\begin{abstract}
We give the explicit expression of the free energy for the Gaussian model and the spherical model on a fully frustrated simple cubic lattice in all dimensions, and compare the results with the ferromagnetic case. For the spherical model, the critical temperature is lower by a factor 2 than the temperature predicted by the usual mean field theory, whereas the Bethe approximation does not predict any transition. We also study the high dimension expansion of the free energy, and conclude that this expansion may hide the transitions. These results show that some difficulties encountered in models of spin glasses also appear in models with non-random interactions.
\end{abstract}

Spin models on fully frustrated lattices generalize the antiferromagnetic triangular lattice [1] and the Villain odd model [2]. In a previous work [3], we have studied the zero temperature properties of Ising spins on fully frustrated simple cubic lattices of any dimension. By comparing our results with those of Alexander and Pincus [4], who studied the case of f.c.c. lattices, it becomes tempting to believe that some properties do not depend on the lattice : the ground state energy per spin always behaves like $-\sqrt{z / 2}$ when the number of neighbours $z$ becomes large.

The study of fully frustrated models is a step in the study of spin glass models. Most of the latter models describe an assembly of spins interacting through bonds of random strength and random sign. One main difficulty however is that the interactions are

(*) Present address : S.P.T., CEN Saclay, 91190 Gif sur Yvette, France. quenched variables, and that it is necessary to average the free energy and not the partition function. To overcome this problem, many authors [5] have used the replica method, but difficulties arise in the limit where the number of replicas is made to vanish $[6,7,8]$.

Fully frustrated models keep one of the main properties of spin glass models : the frustration [9] which represents the competition between the different interactions of the system. But the interactions are no longer random variables and they can be arranged regularly on the lattice. This makes the problem of computing the free energy closer to standard treatments in the models of ferromagnetism. Thus, as explained below, it is possible to compute the exact free energy for the Gaussian model and for the spherical model, by reproducing the calculations of Berlin and $\mathrm{Kac}[10]$ in the case of fully frustrated simple cubic lattices. In spite of the regular arrangement of the interactions on the lattice, we find for this model some characteristic properties of the usual spin glass models : the ground state may have a large dege- 
neracy [3, 4], the usual mean field theory is no longer valid in the limit of infinite dimensionality, where it should be, the existence of a transition can be hidden in the high temperature and high dimension expansions.

In this paper, we start by defining the model, and by extending the results which we gave in our previous work for Ising spins to the case of spins with $n$ components. Afterwards, we give the expressions of the free energy for the Gaussian and the spherical models, and we compare these expressions with those obtained [10] for a ferromagnetic lattice. The critical temperature of the spherical model is lower by a factor 2 than the temperature given by the simplest mean field equations. We also look at the high temperature and high dimension expansions which do not reveal any transition. These two results are very similar to what happens in the Sherrington-Kirkpatrick model of a spin glass [5]. A big difference is that the Bethe method, which was found to give some satisfactory results (the TAP equations) for the Sherrington-Kirkpatrick model [11, 12], does not give any transition and thus is found to be seriously in error for the present problem.

1. The construction of the lattice. - We consider a Hamiltonian of spins located on a lattice

$$
\mathscr{H}=-\sum_{\langle i j\rangle} J_{i j} \mathbf{S}_{\mathbf{i}} \cdot \mathbf{S}_{\mathbf{j}}
$$

The $J_{i j}$ may have two values $+J$ (ferromagnetic bond) or - $J$ (antiferromagnetic bond).

The lattice is fully frustrated if each plaquette (elementary loop) is frustrated, that is to say, if the product of the bonds surrounding any plaquette is negative. Alexander and Pincus [4] have studied the case of the f.c.c. lattices for which plaquettes are triangles. When all the bonds are negative, these lattices are fully frustrated. For a simple lattice, the elementary plaquettes are squares, and the realization of a fully frustrated lattice is more complicated but still possible.

We explained in our previous paper [3] how one can construct a fully frustrated simple cubic lattice in any dimension. For completeness, we recall briefly that this can be done in two steps :

- First, we notice that a fully frustrated lattice can be obtained from a fully frustrated unit hypercube by successive reflexions with respect to the planes limiting the hypercube. So the problem is reduced to building a fully frustrated configuration for the unit hypercube $\mathrm{H}_{d}$.

- Then, we construct the unit hypercube $\mathrm{H}_{d}$ by recurrence : a frustrated square is easily obtained by taking three positive bonds and one negative bond. Now a hypercube $\mathrm{H}_{d}$ in dimension $d$ is built from two $\mathrm{H}_{d-1}$ by drawing bonds between their corresponding sites. By choosing for one $\mathrm{H}_{d-1}$ a configuration of bonds which fully frustrates it, for the other $\mathrm{H}_{d-1}$ the same configuration of bonds, but with all the bond signs interchanged and all the bonds linking the two $\mathrm{H}_{d-1}$ as positive, one obtains a fully frustrated configuration for the hypercube $\mathrm{H}_{d}$.

This is of course not the only way to construct a fully frustrated lattice, but they are all equivalent through gauge transformations [9].

2. Ground state energy. - We consider a model of spins with $n$ components located on the sites of a fully frustrated simple cubic lattice. The problem of finding the ground state energy can be treated by looking at the ground state of the unit hypercube. Here also, a ground state of the whole lattice can be obtained from the ground state of the unit hypercube by reflexions with respect to the planes limiting the hypercube.

The hypercube $\mathrm{H}_{d}$ consists of $2^{d}$ sites. For any configuration of spins, the field $\mathbf{h}_{i}$ created on the site $i$ by its neighbours in the hypercube is given by :

$$
\mathbf{h}_{i}=\sum_{j} J_{i j} \mathbf{S}_{j}
$$

One has :

$$
\mathbf{h}_{i}^{2}=d J^{2}+2 \sum_{j<k} J_{i j} J_{i k} \mathbf{S}_{j} \cdot \mathbf{S}_{k}
$$

By summing this relation over all the spins of the hypercube, one obtains :

$$
\sum_{i=1}^{2^{d}} \mathbf{h}_{i}^{2}=2^{d} d J^{2} .
$$

There is a cancellation of all the crossed terms : in the sum the scalar product $\mathbf{S}_{j} \cdot \mathbf{S}_{k}$ appears twice, the spins $j$ and $k$ are always located on the diagonal of a plaquette and the fact that the plaquette is frustrated implies that the two contributions cancel out.

This relation can be written :

$$
\overline{\mathbf{h}}_{i}^{2}=d J^{2} \text {. }
$$

This equality is true for any configuration of the spins. It can be used to find a lower bound for the energy $E_{0}$ (per spin) of the ground state of the whole lattice

$$
E_{0} \geqslant-\sqrt{d} J
$$

This bound is independent of the number $n$ of components of the spins. It comes from the fact that we want to make $\mid \overline{\mathbf{h} \mid}$ maximum with the constraint that $\overline{\mathbf{h}}^{2}=d J^{2}$. This lower bound is reached when all the spins of the hypercube feel a field whose amplitude is $\sqrt{d} J$

- In the case of Ising spins [3], one can improve the lower bound when the dimension is not a square integer : using the fact that all the fields $h_{i}$ are integers, this bound is obtained by searching for the field distribution with integer values, which makes $\sum\left|h_{i}\right|$ maximum with the constraint $\sum h_{i}^{2}=2^{d} d J^{2}$. 
- For planar spins, we could verify that the bound $-\sqrt{d} J$ is in fact the true ground state energy when $d \leqslant 5$. It is possible that this bound always gives the ground state energy, but we did not succeed in proving it for $d>5$.

- One can show that when the number of components of the spins is large enough $\left(n \geqslant 2^{d-1}\right)$, the ground state energy is $-\sqrt{d} J$. Let us divide the $2^{d}$ sites of the hypercube into two sets : I and II, such that all the sites of one set have all their neighbours in the other set. A ground state is obtained by taking all the spins of set I orthogonal among themselves, and all the spins of set II pointing in the field created by the spins of set I. This insures that the spins of set II are also orthogonal among themselves and one can easily verify that for such a configuration, all the fields $\mathbf{h}_{i}$ have the amplitude $\sqrt{d} J$. This proves that the ground state energy is $-\sqrt{d} J$. The condition $\left(n \geqslant 2^{d-1}\right)$ is actually too restrictive. However, this argument shows that by increasing the number of components of the spins, it is always possible to reach the lower bound for the ground state energy given in (3).

3. The Gaussian model. - Since we can construct a fully frustrated lattice with a periodic configuration of positive and negative bonds, we can derive rigorously the partition function and the free energy of the Gaussian model.

The calculations are given in the appendix. They reproduce, in the case of fully frustrated lattices, those of Berlin and $\mathrm{Kac}$ [10].

One finds for the free energy $f$ per spin

$$
\begin{aligned}
-\frac{f}{T}= & \frac{1}{N} \ln Z=-\frac{1}{4} \frac{1}{(2 \pi)^{d}} \times \\
& \times \int_{0}^{2 \pi} \cdots \int_{0}^{2 \pi} \mathrm{d} \omega_{1} \ldots \mathrm{d} \omega_{d} \\
& \times \ln \left(1-4 K^{2}\left(\cos ^{2} \omega_{1}+\cdots+\cos ^{2} \omega_{d}\right)\right)
\end{aligned}
$$

where $K=\frac{\left|J_{i j}\right|}{T}=\frac{J}{T}$ and $T$ is the temperature.

The critical temperature is the temperature where the free energy (4) becomes singular :

$$
K_{\mathrm{c}}=\frac{J}{T_{\mathrm{c}}}=\frac{1}{2 \sqrt{d}}
$$

The free energy (4) is a function of the two variables $K$ and $d$. It is interesting to note that a high dimension expansion does not lead to the true critical temperature. By expanding (4), one finds

$$
\begin{aligned}
-\frac{f}{T}=-\frac{1}{4} \ln (1-x)+\frac{1}{d} & \frac{1}{16} \frac{x^{2}}{(1-x)^{2}}+\frac{1}{d^{2}} \times \\
& \times \frac{x^{3}(3 x-2)}{64(1-x)^{4}}+0\left(\frac{1}{d^{3}}\right)
\end{aligned}
$$

where $x=2 K^{2} d$.

All the terms of this series are singular when $x=1$, but the true critical point corresponds to the value $x=\frac{1}{2}$ which does not appear in any term of the expansion. This is not very surprising because formula (4) gives a singular part for the free energy which is

$$
\begin{aligned}
\left(T-T_{\mathrm{c}}\right)^{d / 2} \ln (T & \left.-T_{\mathrm{c}}\right)= \\
& =\exp \left(\frac{d}{2} \ln \left(T-T_{\mathrm{c}}\right)\right)^{\prime} \ln \left(T-T_{\mathrm{c}}\right) .
\end{aligned}
$$

This singular part is exponential and so cannot appear in a $1 / d$ expansion.

\section{REMARKS :}

1) In the simple cubic lattice, the number of neighbours $z$ of a given site is $2 d$. We conclude here that a $1 / z$ expansion does not give the true critical temperature. This is rather similar to what happens in the Sherrington-Kirkpatrick model [5] for which the leading term in the $1 / z$ expansion is not singular at $T_{\mathrm{c}}$.

2) Using the calculations given in the appendix, one can easily check that the largest eigenvalue of the matrix $J_{i j}$, which is $2 \sqrt{d} J$, gives the critical temperature as it should.

3) For comparison, we reproduce the expression of the free energy of the ferromagnetic Gaussian model obtained by Berlin and Kac [10] :

$$
\begin{aligned}
-\frac{f}{T}=-\frac{1}{2} & \frac{1}{(2 \pi)^{d}} \int_{0}^{2 \pi} \cdots \int_{0}^{2 \pi} \mathrm{d} \omega_{1} \ldots \mathrm{d} \omega_{d} \times \\
& \times \ln \left(1-2 K\left(\cos \omega_{1}+\cdots+\cos \omega_{d}\right)\right)
\end{aligned}
$$

(for this model all the bonds are positive :

$$
\left.J_{i j}=J=K T\right) .
$$

The critical temperature $T_{\mathrm{c}}^{\prime}$ equals $2 d J$ and the singular part in the free energy has the same form as for the frustrated system.

4. The spherical model. - The Gaussian model is not defined below the critical temperature. Using a Legendre transform, one obtains the spherical model [10] for which the only constraint is

$$
\sum_{i=1}^{N} S_{i}^{2}=N
$$

This model is defined for all temperatures.

The expression for the free energy of the spherical model on a fully frustrated cubic lattice is derived in the appendix ; the result is :

$$
-\frac{f}{T}=-\frac{1}{2}(\ln 2+1)+z-\frac{1}{4} \frac{1}{(2 \pi)^{d}} \int_{0}^{2 \pi} \cdots \int_{0}^{2 \pi} \mathrm{d} \omega_{1} \ldots \mathrm{d} \omega_{d} \ln \left(z^{2}-K^{2}\left(\cos ^{2} \omega_{1}+\cdots+\cos ^{2} \omega_{d}\right)\right)
$$


where the parameter $z$ is given by :

$$
\frac{1}{2} \frac{1}{(2 \pi)^{d}} \int_{0}^{2 \pi} \cdots \int_{0}^{2 \pi} \mathrm{d} \omega_{1} \ldots \mathrm{d} \omega_{d} \frac{z}{z^{2}-K^{2}\left(\cos ^{2} \omega_{1}+\cdots+\cos ^{2} \omega_{d}\right)}=1, \quad \text { if } \quad K<K_{\mathrm{c}}
$$

or

$$
z=\sqrt{d} K \quad \text {, if } K>K_{\mathrm{c}}
$$

and the critical value $K_{\mathrm{c}}$ is given by :

$$
\frac{J}{T_{\mathrm{c}}}=K_{\mathrm{c}}=\frac{1}{2} \frac{\sqrt{d}}{(2 \pi)^{d}} \int_{0}^{2 \pi} \cdots \int_{0}^{2 \pi} \frac{\mathrm{d} \omega_{1} \ldots \mathrm{d} \omega_{d}}{d-\left(\cos ^{2} \omega_{1}+\cdots+\cos ^{2} \omega_{d}\right)} .
$$

To compare this model with the ferromagnetic spherical model, we give the expression of the free energy in the latter case. (By convention, we shall write the quantities in the ferromagnetic case with a prime index.)

$$
-\frac{f^{\prime}}{T}=z^{\prime}-\frac{1}{2}(\ln 2+1)-\frac{1}{2} \frac{1}{(2 \pi)^{d}} \int_{0}^{2 \pi} \cdots \int_{0}^{2 \pi} \mathrm{d} \omega_{1} \ldots \mathrm{d} \omega_{d} \ln \left(z^{\prime}-K\left(\cos \omega_{1}+\cdots+\cos \omega_{d}\right)\right)
$$

where

$$
K_{\mathrm{c}}^{\prime}=\frac{1}{2} \frac{1}{(2 \pi)^{d}} \int_{0}^{2 \pi} \cdots \int_{0}^{2 \pi} \mathrm{d} \omega_{1} \ldots \mathrm{d} \omega_{d} \frac{1}{d-\left(\cos \omega_{1}+\cdots+\cos \omega_{d}\right)}
$$

and

or $z^{\prime}$ given by $\quad 1=\frac{1}{2(2 \pi)^{d}} \int_{0}^{2 \pi} \cdots \int_{0}^{2 \pi} \frac{\mathrm{d} \omega_{1} \ldots \mathrm{d} \omega_{d}}{z^{\prime}-K\left(\cos \omega_{1}+\cdots+\cos \omega_{d}\right)}$

$$
z^{\prime}=d K \quad \text { if } \quad K>K_{\mathrm{c}}^{\prime}
$$

if $K<K_{\mathrm{c}}^{\prime}$.

Using the expressions of the free energy in the two models, we can make three remarks :

1) Both models have no transition at finite $T$ in dimensions 1 or 2 (the integrals (9) and (11) give $\left.K_{\mathrm{c}}=K_{\mathrm{c}}^{\prime}=\infty\right)$. For higher dimensions $(d \geqslant 3)$, the two models have a second order phase transition. This can be seen by showing that the energy is a continuous function of the temperature. So the two models have the same kind of behaviour in all dimensions.

2) The second remark concerns the difference of entropy between the two models in the low temperature phase. Using (6) and (10), it is easy to show that for both models, the entropy goes to $-\infty$ when $T \rightarrow 0$. This is not surprising because the spins are continuous classical variables. The interesting point is that the difference of entropy per spin between the two models is finite and is constant in the low temperature phase :

$$
\begin{aligned}
S-S^{\prime}=\frac{\ln 2}{4}+ & \frac{1}{4(2 \pi)^{d}} \int_{0}^{2 \pi} \cdots \int_{0}^{2 \pi} \mathrm{d} \omega_{1} \ldots \mathrm{d} \omega_{d} \times \\
& \times \ln \left(d-\left(\cos \omega_{1}+\cdots+\cos \omega_{d}\right)\right)
\end{aligned}
$$

which behaves for large $d$ like :

$$
S-S^{\prime} \sim \frac{\ln 2 d}{4}
$$

3) There exists a simple relation between the critical temperatures of the two models : using (9) and (11), it is easy to show that :

$$
K_{\mathrm{c}}=2 \sqrt{d} K_{\mathrm{c}}^{\prime} .
$$

This can be written as :

$$
T_{\mathrm{c}}=T_{\mathrm{c}}^{\prime} / 2 \sqrt{d} .
$$

A high dimension expansion of the critical temperature of the spherical model on the fully frustrated lattice gives :

$$
T_{\mathrm{c}}=J\left(\sqrt{d}-\frac{1}{2 \sqrt{d}}+0\left(d^{-3 / 2}\right)\right)
$$

The critical temperature is lower by a factor 2 than the largest eigenvalue of the matrix $J_{i j}$ in the limit $d \rightarrow \infty$. This was also the case in the spherical model studied by Kosterlitz et al. [12] and in the SherringtonKirkpatrick model $[5,11]$. In order to explain this factor 2 for those models, it was found necessary to use an improved mean field theory, namely the Bethe approximation. We shall see in the last section that this method does not seem well-fitted here. Before though, we are going to analyse the high dimension expansions for the free energy. 
5. High dimension expansions. - 5.1 SPHERICAL MODEL. - As we did for the Gaussian model, it is possible to obtain a high dimension expansion for the free energy of the spherical model using the expression given in $(6,7)$. This expansion is done in the limit $d \rightarrow \infty$ with the constraint that the variable $x=K^{2} d$ is held fixed. The result is :

$$
\begin{array}{r}
-\frac{f}{T}=\frac{\sqrt{1+8 x}-1}{4}-\frac{1}{4} \ln \left(\frac{1+\sqrt{1+8 x}}{2}\right)+ \\
+0\left(\frac{1}{d}\right)
\end{array}
$$

In (15), we give only the leading term. We notice that there is no singularity for positive values of $x$. The only singularities are a branch point for $x=-1 / 8$ (which corresponds to imaginary values of the temperature), and a logarithmic singularity for $x=0$ in the second Riemann sheet. It can also be proved that these are the only singularities which appear in the other terms of the expansion. We conclude that in this problem, the critical temperature which occurs for $x=1$ is hidden by the $1 / d$ expansion. We can only propose a heuristic argument to recover this value : equation (15) contains a logarithm and the series whose sum is this logarithm is convergent only when its argument is less than 2. This value 2 for $\frac{1+\sqrt{1+8 x}}{2}$ gives $x=1$.

5.2 SPINS WITH $n$ COMPONENTS. - We consider now the case of spins with $n$ components. From the Hamiltonian (1), it is possible to expand the free energy in powers of $1 / T$.

If $K=\left|J_{i j}\right| / T$, the free energy $f$ per spin is given by a series :

$$
-\frac{f}{T}=\sum_{p=1}^{\infty} K^{2 p} \alpha_{p}(d, n) .
$$

The coefficients $\alpha_{p}$ are functions of the dimension $d$ of the lattice and of the number $n$ of components of the spins. Using the fact that the lattice is a simple cubic lattice, one can show that the coefficient $\alpha_{p}$ is a polynomial of degree $p$ in the variable $d$. We give here these coefficients up to order $p=3$ for fully frustrated cubic lattices in dimension $d$ :

$$
\begin{aligned}
-\frac{f}{T}= & K^{2} \frac{d}{2 n}+K^{4}\left(-\frac{d^{2}}{2 n^{3}}+\frac{d}{8 n(n+2)}-\frac{d}{8 n^{2}}+\frac{d}{2 n^{3}}\right)+ \\
& +K^{6}\left(\frac{4 d^{3}}{3 n^{5}}-\frac{d^{2}}{n^{3}(n+2)}+\frac{d^{2}}{n^{4}}-\frac{3 d^{2}}{n^{3}}+\frac{d}{48 n(n+2)(n+4)}+\frac{d}{n^{3}(n+2)}-\frac{d}{16 n^{2}(n+2)}+\frac{d}{24 n^{3}}-\frac{d}{n^{4}}+\frac{5 d}{3 n^{5}}\right) \\
& +0\left(K^{8}\right) .
\end{aligned}
$$

As the highest degree terms in $\alpha_{p}$ are proportional to $d^{p}$, the free energy given in (16) can be written as :

$$
-\frac{f}{T}=A_{0}\left(K^{2} d\right)+\frac{1}{d} A_{1}\left(K^{2} d\right)+0\left(\frac{1}{d^{2}}\right)
$$

where $A_{i}$ is the series obtained by keeping only terms of degree $d^{p-i}$ in $\alpha_{p}$.

One can prove that the highest degree term of $\alpha_{p}$ is always of the form :

$$
\beta_{p} \frac{d^{p}}{n^{2 p-1}}
$$

where $\beta_{p}$ is a number which does not depend on $d$ or $n$. As it can be seen in (17), the other terms are more complicated functions of $n$. In the limit $n \rightarrow \infty$, one recovers the spherical model :

$$
\left\{\begin{array}{l}
n \rightarrow \infty \\
\frac{K}{n} \rightarrow K_{0} \\
\frac{1}{n} f(n, K) \rightarrow f\left(K_{0}\right) \text { the free energy of the spherical } \\
\text { model } .
\end{array}\right.
$$

Because of the simple $n$ dependence of the leading terms in $\alpha_{p}$, it is possible to find explicitly the function $A_{0}\left(K^{2} d\right)$ of the expansion (18)

$$
\begin{aligned}
-\frac{f}{T} & =A_{0}\left(K^{2} d\right)+0\left(\frac{1}{d}\right) \\
& =n\left(\frac{\sqrt{1+8 K^{2} d / n^{2}}-1}{4}-\frac{1}{4} \times\right. \\
& \left.\times \ln \left(\frac{1+\sqrt{1+8 K^{2} d / n^{2}}}{2}\right)\right)+0\left(\frac{1}{d}\right) .
\end{aligned}
$$

\section{REMARKS :}

1) We have used the fact that in the limit $n \rightarrow \infty$, one recovers the spherical model. This was proved by Stanley [13] in the ferromagnetic case but we think this remains true for fully frustrated lattices. We verified it for the first terms of the high temperature expansion.

2) This method of expanding the free energy or other quantities in powers of $1 / d$ has often been used [14]. For the ferromagnetic Ising model, one can obtain the $1 / d$ expansion of the critical temperature using the $1 / T$ expansion of the susceptibility. It has 
been shown recently [15] however that for the ferromagnetic Ising model, the series obtained by keeping only the highest powers of $d$ in the free energy has a zero radius of convergence. Our result given in equation (20) exhibits an important difference between the ferromagnetic case and the fully frustrated case for which the radius of convergence is finite.

3) As said earlier, in Section 5, the expression (20) does not give any information about the existence of a transition.

6. Mean field theories. - The high dimension limit is interesting because one expects that some sort of mean field theory becomes exact in this limit. In this last section, we are going to use the usual mean field theory and the Bethe approximation. We shall see that none of them yields the critical temperature of the spherical model.

To simplify our notations, let us introduce two functions $Z_{n}(\mathbf{h})$ and $A_{n}(\mathbf{h})$.

$$
\begin{aligned}
Z_{n}(\mathbf{h}) & =\int_{\mathbf{S}^{2}=1} \mathrm{~d}^{n} \mathbf{S} \exp (\mathbf{h} . \mathbf{S}) \\
& =Z_{n}(0)|h|^{1-\frac{n}{2}} I_{\frac{n}{2}-1}(|h|) \\
\mathbf{A}_{n}(\mathbf{h}) & =\frac{1}{Z_{n}(\mathbf{h})} \frac{\partial Z_{n}(\mathbf{h})}{\partial \mathbf{h}}
\end{aligned}
$$

In (21), the integral is performed over the unit sphere in dimension $n$, and $I_{n}$ is the modified Bessel function [14]. These two functions can be expanded around $\mathbf{h}=0$ :

$$
\begin{aligned}
& Z_{n}(\mathbf{h})=Z_{n}(0)\left[1+\frac{\mathbf{h}^{2}}{2 n}+\frac{\left(\mathbf{h}^{2}\right)^{2}}{8 n(n+2)}+\cdots\right] \\
& \mathbf{A}_{n}(\mathbf{h})=\mathbf{h}\left(\frac{1}{n}-\frac{\mathbf{h}^{2}}{n^{2}(n+2)}+\cdots\right)
\end{aligned}
$$

For a given Hamiltonian like

$$
\mathscr{H}=-\sum_{\langle i j\rangle} J_{i j} \mathbf{S}_{\mathbf{i}} \cdot \mathbf{S}_{\mathbf{j}}
$$

the usual mean field theory consists of assuming that each spin has an average magnetization $\mathbf{m}_{i}$ and of computing this magnetization $\mathbf{m}_{\boldsymbol{i}}$ with the approximate Hamiltonian :

$$
\mathscr{H}^{\prime}=-\sum_{j} J_{i j} \mathbf{m}_{j} \cdot \mathbf{S}_{\mathbf{i}}
$$

When the spins have $n$ components, this theory gives the mean field equations :

$$
\mathbf{m}_{i}=\mathbf{A}_{n}\left(\frac{1}{T} \sum_{j} J_{i j} \mathbf{m}_{j}\right) .
$$

In the high temperature region, the equation (23) have only one solution $\mathbf{m}_{i}=0$. If we look for a seconc order transition, it is enough to linearize the equation (23) and to find the temperature for which the linearized equations have a non-vanishing solution. If we keep in mind that the largest eigenvalue of the matrix $J_{i j}$ is $2 \sqrt{d} J$, we find the critical temperature predicted by this mean field theory :

$$
T_{\mathrm{c}}=\frac{2 \sqrt{d} J}{n} \text {. }
$$

The spherical model can be obtained in the limit $n \rightarrow \infty$ with $\frac{J}{n} \rightarrow J_{0}$ as we noted in (19). So this theory gives $T_{\mathrm{c}}=2 \sqrt{d} J_{0}$ for the spherical model though the true transition temperature is lower by a factor 2 (Eq. (14)). This mean field theory is not adequate here, no more than in the case of the SherringtonKirkpatrick model, where Thouless et al. [11] argued that a proper remedy is to use the Bethe approximation. This approximation consists of assuming that each spin has an average magnetization $\mathbf{m}_{i}$ and of computing this magnetization with the approximate Hamiltonian

$$
\mathcal{H}^{\prime \prime}=-\sum_{j}\left(J_{0 j} \mathbf{S}_{0}+\mathbf{h}_{j}\right) \cdot \mathbf{S}_{\mathbf{j}} .
$$

This Hamiltonian describes a central spin $\mathbf{S}_{0}$ and all its neighbours $\mathbf{S}_{\mathbf{j}}$. The fields $\mathbf{h}_{j}$ represent the effect on the spins $\mathbf{S}_{\mathbf{j}}$ of their other neighbours.

With this approximate Hamiltonian, we obtain $\mathbf{m}_{0}$ and $\mathbf{m}_{j}$ :

$$
\begin{gathered}
\mathbf{m}_{0}=\frac{\int \mathrm{d}^{n} \mathbf{S}_{0} \mathbf{S}_{0} \prod_{i=1}^{z} Z_{n}\left(\frac{\mathbf{h}_{i}}{T}+\frac{J_{0 i}}{T} \mathbf{S}_{0}\right)}{\int \mathrm{d}^{n} \mathbf{S}_{0} \prod_{i=1}^{z} Z_{n}\left(\frac{\mathbf{h}_{i}}{T}+\frac{J_{0 i}}{T} \mathbf{S}_{0}\right)} \\
\mathbf{m}_{j}=\frac{\int \mathrm{d}^{n} \mathbf{S}_{0} \mathbf{A}_{\mathbf{n}}\left(\frac{J_{0 j} \mathbf{S}_{0}+\mathbf{h}_{j}}{T}\right) \prod_{i=1}^{z} Z_{n}\left(\frac{\mathbf{h}_{i}}{T}+\frac{J_{0 i}}{T} \mathbf{S}_{0}\right)}{\int \mathrm{d}^{n} \mathbf{S}_{0} \prod_{i=1}^{z} Z_{n}\left(\frac{\mathbf{h}_{i}}{T}+\frac{J_{0 i}}{T} \mathbf{S}_{0}\right)} .
\end{gathered}
$$

We have $z$ spins $\mathbf{S}_{\mathbf{j}}$ and $(z+1)$ equations. So it is in principle possible to eliminate the fields $\mathbf{h}_{j}$ and to find a relation between $\mathbf{m}_{0}$ and the $\mathbf{m}_{j}$. Since we expect a critical temperature $T_{\mathrm{c}}$ of order $\sqrt{d}$, we have

$$
\left|J_{i j}\right| / T_{\mathrm{c}} \ll 1
$$

and it is possible to put equations (25) in a simpler form which should be valid in the critical region :

$$
\begin{aligned}
& \mathbf{m}_{0}=\mathbf{A}_{\mathbf{n}}\left(\sum_{i=1}^{z} \frac{J_{0 i}}{T} \mathbf{A}_{\mathbf{n}}\left(\frac{\mathbf{h}_{i}}{T}\right)\right) \\
& \mathbf{m}_{j}=\mathbf{A}_{\mathbf{n}}\left(\frac{\mathbf{h}_{j}}{T}+\frac{J_{0 j}}{T} \mathbf{m}_{0}\right) .
\end{aligned}
$$


These equations are obtained from (25) using the fact that :

$$
\begin{aligned}
\prod_{i=1}^{z} Z_{n}\left(\frac{\mathbf{h}_{i}}{T}+\frac{J_{0 i}}{T} \mathbf{S}_{0}\right) \sim & {\left[\prod_{i=1}^{z} Z_{n}\left(\frac{\mathbf{h}_{i}}{T}\right)\right] \times } \\
& \times \exp \left(\sum_{i=1}^{z} \frac{J_{0 i}}{T} \mathbf{S}_{0} \cdot \mathbf{A}_{\mathbf{n}}\left(\frac{\mathbf{h}_{i}}{T}\right)\right) .
\end{aligned}
$$

Here also a second order phase transition is obtained when the linearized equations (26) admit a non-zero solution. If we eliminate the fields $h_{i}$ in the linearized equations, we find :

$$
\mathbf{m}_{0}=\frac{1}{n} \sum_{j=1}^{z} \frac{J_{0 j}}{T} \mathbf{m}_{j}-\frac{1}{n^{2}} \sum_{j=1}^{z} \frac{J_{0 j}^{2}}{T^{2}} \mathbf{m}_{0} .
$$

As the largest eigenvalue of matrix $J_{i j}$ is $2 \sqrt{d} J$, the critical temperature $T_{\mathrm{c}}$ has to verify :

$$
1=\frac{1}{n} \frac{2 \sqrt{d} J}{T_{\mathrm{c}}}-\frac{2 d}{n^{2}} \frac{J^{2}}{T_{\mathrm{c}}^{2}} .
$$

This equation has no real solution; this proves that the Bethe approximation does not give a second order transition.

\section{REMARKS :}

1) In the case of f.c.c. lattices which has been studied by Alexander and Pincus [4], the coordination number $z$ is given by : $z=2 d(d-1)$.

It is also possible to write the predictions of the usual mean field theory and of the Bethe approximation for the f.c.c. lattice. Equation (24) becomes :

$$
T_{\mathrm{c}}=\frac{\lambda}{n} J
$$

and equation (28) :

$$
1=\frac{\lambda}{n} \frac{J}{T_{\mathrm{c}}}-\frac{z}{n^{2}} \frac{J^{2}}{T_{\mathrm{c}}^{2}}
$$

where $\lambda=2(d-1)$ for odd $d$ and $\lambda=2 d$ for even $d$.

The results may then be summarized in a table giving the critical temperature $T_{\mathrm{c}}$ for large $z$ for both lattices :

Ferromagnetic system
Fully frustrated system
model
where the mean field and Bethe results correspond
to the spherical model. The striking fact is that models
and approximations which give equivalent results in
leading order for ferromagnetic systems may yield
very different answers for frustrated systems.

2) The difference between the usual mean field theory and the Bethe approximation is the presence of a second term in the right hand side of equation (27) which takes into account the polarization of the spin $\mathbf{S}_{\mathbf{j}}$ by the effect of the spin $\mathbf{S}_{0}$, as pointed out by Thouless et al. for spin glass models [11]. As we expect here that the critical temperature is proportional to $\sqrt{d}$, this term has to be considered and it leads to different predictions between these two approximations. In the ferromagnetic case, the usual mean field theory predicts the right critical temperature in the limit $d \rightarrow \infty$, and the Bethe approximation gives only corrections which become negligible in this limit.

3) It is much easier to study second order than first order phase transitions using equations (26) : first order phase transitions depend on the non-linear form of functions $\mathbf{A}_{n}$, whereas second order phase transitions depend only on the linear part of these functions. We think that the equations (26) do not

$$
\begin{gathered}
\text { Mean-field } \\
\text { approximation }
\end{gathered}
$$$$
\begin{array}{ll}
\overline{z J} & \overline{z J} \\
\sqrt{z / 2} J & \sqrt{2 z} J
\end{array}
$$

Bethe approximation<smiles>C1C[Te]1</smiles>
no 2 nd order transition

give a first order phase transition for large $d$, but we did not find a rigorous proof of it. Anyway, in the limit $n \rightarrow \infty$, the essential point is that these equations do not give a second order phase transition.

7. Conclusion. - In this work, we have obtained the expression of the free energy for the Gaussian model and for the spherical model when the lattice is fully frustrated. Our main result is that the critical temperature of the spherical model is lower by a factor 2 than the temperature predicted by the usual mean field theory, and that the Bethe approximation is not valid in this problem. Our main conclusion is that the problem of finding a good mean field theory (which becomes exact when the coordination number is large) is still open.

We have also studied the high dimension expansions of the free energy : for the Gaussian model as well as for the spherical model, the transitions are hidden by these expansions.

We have focused this work on the high dimension limit in order to be able to examine critically the mean field theories. For direct comparisons with experiments however, the most important dimensions are 2 and 3. A recent Monte Carlo calculation [16] shows a 
first order phase transition for the f.c.c. antiferromagnetic lattice with Ising spins in dimension 3. It would be interesting to know if a corresponding transition exists for the fully frustrated simple cubic lattice in dimension 3 , and what happens when the number of components of the spins is larger than 1 .

We wish to thank J. M. Drouffe, J. M. Maillard and P. Moussa for many helpful discussions.

\section{APPENDIX}

1. The free energy of the Gaussian model. - The partition function of the Gaussian model is :

$$
Z=\frac{\int \mathrm{d} S_{1} \ldots \mathrm{d} S_{N} \exp \left[-\frac{1}{2} \sum_{i=1}^{N} S_{i}^{2}+\sum_{\langle i j\rangle} \frac{1}{T} J_{i j} S_{i} S_{j}\right]}{\int \mathrm{d} S_{1} \ldots \mathrm{d} S_{N} \exp \left[-\frac{1}{2} \sum_{i=1} S_{i}^{2}\right]}
$$

The interactions $J_{i j}$ take the values $+J$ or $-J$ so as to realize a fully frustrated lattice.

If we define an $N \times N$ matrix $A$ by :

$$
A_{j i}=A_{i j}=\frac{1}{2} \delta_{i j}-\frac{J_{i j}}{2 T}
$$

The partition function is given by :

$$
Z=2^{-N / 2}(\operatorname{det} A)^{-1 / 2} \text {. }
$$

If we take a cubic lattice in dimension $d$ with periodical boundary conditions of size

$$
2 n_{1} \times 2 n_{2} \times \cdots \times 2 n_{d}=N,
$$

we are going to show by recurrence that the eigenvalues of matrix $A$ are :

$$
\begin{aligned}
& -\frac{1}{2}+K \sqrt{\cos ^{2} k_{1}+\cdots+\cos ^{2} k_{d}} \\
& \text { with the degeneracy } 2^{d-1} \\
& -\frac{1}{2}-K \sqrt{\cos ^{2} k_{1}+\cdots+\cos ^{2} k_{d}} \\
& \text { with the degeneracy } 2^{d-1} \\
& \text { where } \quad K=\frac{\left|J_{i j}\right|}{T}, \quad k_{i}=\frac{2 \pi}{2 n_{i}} p_{i}
\end{aligned}
$$

and $p_{i}$ is an integer $0 \leqslant p_{i} \leqslant n_{i}-1$.

It is easy to verify it in dimension 1 , by taking a ferromagnetic chain. Now we assume this is true in dimension $d$, and we are going to prove that it remains true in dimension $d+1$.

Consider again the construction of a fully frustrated lattice in dimension $d+1$ using a lattice of dimension $d$ : a lattice in dimension $d+1$ is an alternate superposition of lattices in dimension $d$ connected by ferromagnetic interactions in the $(d+1)$ th direction. Here, by alternate superposition, we mean that if we take a configuration of interactions which fully frustrate one lattice in dimension $d$, the next lattice (when one moves in the $(d+1)$ th direction) has the configuration obtained by changing the signs of all the inter- actions (this construction also works to go from $d=1$ to $d=2$ ).

Call $V(\mathbf{r})$ an eigenvector of the matrix $A$ for a lattice in dimension $d$ which corresponds to the eigenvalue $\frac{1}{2}+K \sqrt{\cos ^{2} k_{1}+\cdots+\cos ^{2} k_{d}}$. This vector consists of a given number for each site $\mathbf{r}$ of the lattice $\left(1 \leqslant r_{i} \leqslant 2 n_{i}\right)$. In the next lattice of dimension $d$, this vector is an eigenvector with the eigenvalue $\frac{1}{2}-K \sqrt{\cos ^{2} k_{1}+\cdots+\cos ^{2} k_{d}}$.

Consider now the vector $W(\mathbf{r}, x)$ consisting of a given number for each site of the $d+1$ lattice : $x$ is the $(d+1)$ th coordinate of a lattice site, whereas $\mathbf{r}$ represents its $d$ first coordinates. We take for $W$ a vector defined by :

$$
\begin{array}{l|l}
W(\mathbf{r}, x)=\alpha \mathrm{e}^{i k_{d+1} x} V(\mathbf{r}) & \text { if } x \text { is even } \\
W(\mathbf{r}, x)=\beta \mathrm{e}^{i k_{d+1} x} V(\mathbf{r}) & \text { if } x \text { is odd }
\end{array}
$$

with

$$
k_{d+1}=\frac{2 \pi}{2 n_{d+1}} p_{d+1} \text { and } 0 \leqslant p_{d+1} \leqslant n_{d+1}-1 .
$$

If $W^{\prime}=A W$, the vector $W^{\prime}$ is defined like vector $W$ in (A.3) by two numbers $\alpha^{\prime}$ and $\beta^{\prime}$ :

$$
\begin{array}{rl|l}
W^{\prime} & =\alpha^{\prime} \mathrm{e}^{i k_{d+1} x} V(\mathbf{r}) & \text { if } x \text { is even } \\
& =\beta^{\prime} \mathrm{e}^{i k_{d+1} x} V(\mathbf{r}) & \text { if } x \text { is odd }
\end{array}
$$

$\alpha^{\prime}$ and $\beta^{\prime}$ are related to $\alpha$ and $\beta$ by :

$$
\begin{aligned}
\alpha^{\prime}=\left(\frac{1}{2}+\right. & \left.K \sqrt{\cos ^{2} k_{1}+\cdots+\cos ^{2} k_{d}}\right) \alpha+ \\
& +K \cos \left(k_{d+1}\right) \beta \\
\beta^{\prime}=K \cos \left(k_{d+1}\right) \alpha+ & \\
& +\left(\frac{1}{2}-K \sqrt{\cos ^{2} k_{1}+\cdots+\cos ^{2} k_{d+1}}\right) \beta .
\end{aligned}
$$

The diagonalization shows that we obtain the two eigenvalues :

$$
\frac{1}{2} \pm K \sqrt{\cos ^{2} k_{1}+\cdots+\cos ^{2} k_{d+1}} .
$$


This proves the result (A.2). Using (A.1), we find for the partition function :

$$
\begin{aligned}
Z= & \exp \left[-\frac{1}{2} \sum_{p_{1}=1}^{n_{1}} \ldots \sum_{p_{d}=1}^{n_{d}} 2^{d-1} \times\right. \\
& \left.\times \ln \left(1-4 K^{2}\left(\cos ^{2} \frac{\pi p_{1}}{n_{1}}+\cdots+\cos ^{2} \frac{\pi p_{d}}{n_{d}}\right)\right)\right] .
\end{aligned}
$$

This gives in the thermodynamic limit :

$$
\begin{aligned}
\frac{1}{N} \ln Z= & -\frac{1}{4} \frac{1}{(2 \pi)^{d}} \int_{0}^{2 \pi} \ldots \int_{0}^{2 \pi} \mathrm{d} \omega_{1} \ldots \mathrm{d} \omega_{d} \times \\
& \times \ln \left(1-4 K^{2}\left(\cos ^{2} \omega_{1}+\cdots+\cos ^{2} \omega_{d}\right)\right) .
\end{aligned}
$$

2. The free energy of the spherical model. - The partition function is given by :

$$
\begin{aligned}
Z & =\int \mathrm{d} S_{1} \ldots \int \mathrm{d} S_{N} \delta\left(\sum S_{i}^{2}-N\right) \exp \left(\sum_{\langle i j\rangle} \frac{J_{i j}}{T} S_{i} S_{j}\right) \\
& =(\pi)^{N / 2} \frac{1}{2 \pi} \int \mathrm{d} \lambda \mathrm{e}^{i \lambda N}(\operatorname{det} B)^{-1 / 2}
\end{aligned}
$$

where the matrix $B$ is an $N \times N$ matrix defined by :

$$
B_{i j}=B_{j i}=i \lambda \delta_{i j}-\frac{J_{i j}}{2 T} .
$$

One can diagonalize matrix $B$ as we did for matrix $A$ in the case of the Gaussian model.

In the thermodynamic limit $(N \rightarrow \infty)$, one can calculate the integral in the formula (A.4) by a saddle point method. We find

$$
\begin{aligned}
\frac{\ln Z}{N} & =z-\frac{1}{2}(\ln 2+1)-\frac{1}{4} \frac{1}{(2 \pi)^{d}} \times \\
& \times \int_{0}^{2 \pi} \cdots \int_{0}^{2 \pi} \mathrm{d} \omega_{1} \ldots \mathrm{d} \omega_{d} \\
& \times \ln \left(z^{2}-K^{2}\left(\cos ^{2} \omega_{1}+\cdots+\cos ^{2} \omega_{d}\right)\right)
\end{aligned}
$$

where $z$ is the value of $i \lambda$ at the saddle point and is given by (A.6) in the high temperature phase, and by (A.7) in the low temperature phase :

$$
\begin{gathered}
1=\frac{1}{2} \frac{1}{(2 \pi)^{d}} \int_{0}^{2 \pi} \cdots \int_{0}^{2 \pi} \mathrm{d} \omega_{1} \ldots \mathrm{d} \omega_{d} \times \\
\times \frac{z}{z^{2}-K^{2}\left(\cos ^{2} \omega_{1}+\cdots+\cos ^{2} \omega_{d}\right)} \\
z=\sqrt{d} K .
\end{gathered}
$$

The critical temperature corresponds to the value of $K$ for which (A.6) and (A.7) have the same solution :

$$
\begin{aligned}
K_{\mathrm{c}}=\frac{J}{T_{\mathrm{c}}}=\frac{1}{(2 \pi)^{d}} & \frac{1}{2} \int_{0}^{2 \pi} \cdots \int_{0}^{2 \pi} \mathrm{d} \omega_{1} \ldots \mathrm{d} \omega_{d} \times \\
& \times \frac{\sqrt{d}}{d-\left(\cos ^{2} \omega_{1}+\cdots+\cos ^{2} \omega_{d}\right)} .
\end{aligned}
$$

REMARK. - It is always possible to add a constant to the free energy. In (A.5), we have chosen this constant such that the free energy is zero when the temperature goes to infinity.

\section{References}

[1] Wannier, G., Phys. Rev. 79 (1950) 357.

[2] Villain, J., J. Phys. C 10 (1977) 1717.

[3] Derrida, B., Pomeau, Y., Toulouse, G., Vannimenus, J., J. Physique 40 (1979) 617

[4] Alexander, S., Pincus, P., J. Phys. A 13 (1980) 263.

[5] Sherrington, D., KirkPatrick, S., Phys. Rev. Lett. 35 (1975) 1792.

Kirkpatrick, S., Sherrington, D., Phys. Rev. B 17 (1978) 4384.

[6] De Almeida, J. R. L., Thouless, D. J., J. Phys. A 11 (1978) 983.

[7] Blandin, A., Gabay, M., Garel, T. (1979) preprint.

[8] BRAY, A. J., MOORE, M. A. (1979) preprint.

[9] Toulouse, G., Comm. on Phys. 2 (1977) 115.
Vannimenus, J., Toulouse, G., J. Phys. C 10 (1977) L-537.

[10] Berlin, T. H., KaC, M., Phys. Rev. 86 (1952) 821.

[11] Thouless, D. J., Anderson, P. W., Palmer, R. G., Philos. Mag. 35 (1977) 593.

[12] Kosterlitz, J. M., Thouless, D. J., Jones, R. C., Phys. Rev. Lett. 36 (1976) 1217.

[13] Stanley, H. E., Phys. Rev. 176 (1968) 718.

[14] Fischer, M. E., Gaunt, D. S., Phys. Rev. 133 (1964) A-224.

ABE, R., Prog. Theor. Phys. 47 (1972) 62.

Balian, R., Drouffe, J. M., Itzýrson, C., Phys. Rev. D 11 (1975) 2104.

[15] Drouffe, J. M., Parisi, G., Sourlas, N. (preprint 1979).

[16] Phani, M. K., Lebowitz, J. L., Kalos, M. H., Tsai, C. C., Phys. Rev. Lett. 42 (1979) 577. 